ICMPA-MPA/2008/12

January 17,2021

\title{
Noncommutative complex Grosse-Wulkenhaar model
}

\author{
Mahouton Norbert Hounkonnou and Dine Ousmane Samary
}

\begin{abstract}
This paper stands for an application of the noncommutative (NC) Noether theorem, given in our previous work [AIP Proc 956 (2007) 55-60], for the NC complex Grosse-Wulkenhaar model. It provides with an extension of a recent work [Physics Letters B 653 (2007) 343-345]. The local conservation of energy-momentum tensors (EMTs) is recovered using improvement procedures based on Moyal algebraic techniques. Broken dilatation symmetry is discussed. NC gauge currents are also explicitly computed.
\end{abstract}

Keywords. Noncommutative field theory, Moyal algebra, Noether theorem, Ward identity operator, Energy-momentum tensor.

\section{Introduction}

Field theory on noncommutative geometry, or noncommutative field theory (NCFT), for which there exists a respectable body of mathematical and physical knowledge (see [1]-[15] and references therein), is most often performed over a Moyal space $\mathrm{R}_{\theta}^{D}$, a deformed $\mathrm{D}$-dimensional space endowed with a constant Moyal $\star$-bracket of coordinate functions

$$
\left[x^{\mu}, x^{\nu}\right]_{\star}=i \Theta^{\mu \nu}
$$

where $\Theta$ is a $D \times D$ non-degenerate skew-symmetric matrix (which requires $\mathrm{D}$ even), usually chosen in canonical form as

$$
\Theta=\theta J \text { with } J=\left(\begin{array}{ll}
0 & I_{N} \\
-I_{N} & 0
\end{array}\right) .
$$

] $0,+\infty\left[\ni \theta\right.$ is a square length dimensional parameter, $\left([\theta]=[L]^{2}\right)$. The corresponding product of functions is the associative, noncommutative MoyalGroenewold-Weyl product, simply called hereafter Moyal product or $\star$-product defined by

$$
(f \star g)(x)=\mathrm{m}\left\{e^{i \frac{\Theta^{\rho \sigma}}{2} \partial_{\rho} \otimes \partial_{\sigma}} f(x) \otimes g(x)\right\}, \quad x \in \mathbf{R}_{\theta}^{D}, \quad \forall f, g \in \mathcal{S}\left(\mathbf{R}_{\theta}^{D}\right)(1.3)
$$

CP1079, Geometric Methods in PhysicsProceedings of the XXVII Workshop on Geometric Methods in Physics edited by P. Kielanowski, A. Odzijewicz, M. Schlichenmaier, and T. Voronov O 2008 American Institute of Physics 978-0-7354-0610-0/08/. 
$\mathrm{m}$ is the ordinary multiplication of functions and $\mathcal{S}\left(\mathrm{R}_{\theta}^{D}\right)$ - the space of suitable Schwartzian functions. For precise definition, see [11]-[14]. Such a noncommutative geometry possesses the specific pathology to break both the Lorentz invariance by the presence of $\Theta^{\mu \nu}$, as $\left[x^{\mu}, x^{\nu}\right]_{\star}=i \Theta^{\mu \nu}$ is not generally invariant under rotation, and the local character of the theory thanks to the property that

$$
(f \star g)(z)=\int d^{D} x d^{D} y K(x, y ; z) f(x) f(y)
$$

is evaluated through a two-point kernel

$$
K(x, y ; z)=\delta(z-x) \star \delta(z-y)=\frac{1}{(2 \pi)^{D} \operatorname{det} \Theta} e^{i(z-x) \Theta^{-1}(x-y)} .
$$

Besides, the uncertainty principle is expressed by $\Delta x \Delta y \geq \theta$. For more details on the NCFT characterization, see [11] and references therein. Peculiar features of noncommutative field theories (NCFT) in the Moyal $\star$-product description generally engender energy momentum tensors (EMTs) which are not locally conserved, not traceless in the massless situation and, not symmetric and not gauge invariant in gauge theories [2, 4, 3, 5, 10, 11.

Noether's procedure for infinitesimal translation symmetry in noncommutative (NC) scalar field theory has been studied by Gerhold et al. 2 and by Abou-Zeid and Dorn [3. According to their works, the canonical EMT appears to be symmetric albeit not locally conserved. From algebraic techniques, it can be obtained an improved locally conserved EMT [3. Nevertheless, this improved tensor fails to be symmetric. It is noteworthy that, in classical field theory, a symmetric EMT is related to a locally conserved angular momentum. Further, in NCFT, the constant tensor $\Theta^{\mu \nu}=-i\left[x^{\mu}, x^{\nu}\right]$, characterizing the noncommutative geometry, is not a Lorentz tensor and obviously breaks the Lorentz invariance. Therefore, the nonlocal conservation of angular momentum and then the asymmetry of EMT are a priori allowed.

$\mathrm{NC}$ versions of Noether currents have been worked out for translation, dilatation and local gauge symmetries [2]-7].

So far, all these studies have been performed before the advent of the new class of renormalizable NC field theories (NCRFT) built on the Grosse and Wulkenhaar $\phi^{4}$ scalar field theory [9, 14].

In our previous work [11, an explicit formulation of an analogue of the Noether theorem in Moyal NCFTs has been provided at the operator level as follows. Given $W$ the global canonical NC Ward identity operator (WIop) for a transformation, then the following statement holds:

Theorem (NC Noether theorem, Ben Geloun and Hounkonnou): If a $N C$ action $S$ is invariant under a set of transformations, then

$$
W S=-\int d^{D} x \operatorname{Tr} \partial^{\mu} J_{\mu}=0,
$$

and there exists a globally conserved vector current $J_{\mu}$.

Albeit the local conservation of the current is not required, many facts invite us to consider this statement as actually the best of what we can 
expect from the $\mathrm{NC}$ action formulation. Indeed, $(i)$ it is the $\mathrm{NC}$ method the closest of usual Noether procedure; $(i i)$ in NC space-space $\theta^{0 i}=0$ geometry, there exists an exactly conserved $D$-vector momentum $P_{\mu}$ for infinitesimal translation symmetry which readily generalizes its undeformed counterpart; (iii) it can be proved that regularization procedures improve quantities to recover local conservation property for many field theories and $(i v)$ finally, at the parameter limit $\theta \rightarrow 0$ the globally conserved currents obtained through the NC WIop method reduce to classical Noether currents.

Recently, this statement has been successfully applied to investigate EMTs in renormalizable noncommutative scalar field theory [10].

The purpose of this paper is to give, as another original contribution, an application of the above NC Noether theorem in the framework of the NC complex Grosse-Wulkenhaar (GW) model, to perform the local conservation of energy-momentum tensors (EMTs), using improvement procedures based on Moyal algebraic techniques, as well as to analyze the broken dilatation symmetry and to explicitly compute NC gauge currents.

\section{Noncommutative complex GW model}

The NC complex Grosse-Wulkenhaar Lagrangian action can be written as:

$$
\begin{aligned}
S_{\star}^{\Omega}[\phi, \bar{\phi}] & =\int d^{D} x\left[\partial_{\mu} \phi \star \partial^{\mu} \bar{\phi}+m^{2} \phi \star \bar{\phi}+\frac{\Omega^{2}}{2}\left(\tilde{x}_{\mu} \phi\right) \star\left(\tilde{x}^{\mu} \bar{\phi}\right)\right. \\
& \left.+\frac{\lambda}{2.4 !}(\phi \star \bar{\phi} \star \phi \star \bar{\phi}+\phi \star \bar{\phi} \star \bar{\phi} \star \phi)\right]
\end{aligned}
$$

where $\widetilde{x}=2\left(\Theta^{-1}\right) \cdot x, \Theta$ breaks into diagonal blocks $\left(\begin{array}{ll}0 & \theta \\ -\theta & 0\end{array}\right) \cdot \phi$ is a complex scalar field (with rapid decay). The harmonic term $\Omega$ ensures ultraviolet (UV)/infrared (IR) freedom for the action implying its renormalizability, and such that the Lagrangian action becomes covariant under Langmann-Szabo duality [15], i.e. covariant under the symmetry: $\tilde{x}_{\mu} \longleftrightarrow p_{\mu} \equiv \partial_{\mu}$. The Lagrangian density depending explicitly on $x^{\mu}$, through the field $\phi$ interaction with a harmonic external source, does not describe a closed system. Furtheremore, it is not invariant under space-time translation. Besides, at the parameter limit $\theta \rightarrow 0$, the model does not converge to the ordinary $\phi^{4}$ scalar field theory due to the presence of the inverse matrix $\left(\Theta^{-1}\right)$, then causing a singularity. The $\star$-Grosse-Wulkenhaar $\phi_{D}^{4}$ theory is renormalizable at all orders in $\lambda$. This result has been now proved by various methods (see [14] and references therein). It is a matter of algebra to recast the Grosse-Wulkenhaar harmonic term as

$$
\begin{aligned}
\left(\tilde{x}_{\mu} \phi\right) \star\left(\tilde{x}^{\mu} \bar{\phi}\right) & =\frac{1}{4}\left(\tilde{x}_{\mu} \star \phi \star \tilde{x}^{\mu} \star \bar{\phi}+\tilde{x}_{\mu} \star \phi \star \bar{\phi} \star \tilde{x}^{\mu}\right. \\
& \left.+\phi \star \tilde{x}_{\mu} \star \tilde{x}^{\mu} \star \bar{\phi}+\phi \star \tilde{x}_{\mu} \star \bar{\phi} \star \tilde{x}^{\mu}\right)
\end{aligned}
$$

and to re-express accordingly the action (2.1) so that it now entirely lies in the $\star$-algebra of fields with the advantage to be stable under formal $\star$-algebraic 
computations (such that the cyclicity of $\star$-factors under integral). We get the equations of motion for $\phi$ and $\bar{\phi}$ as follows:

$$
\begin{aligned}
\frac{\delta S_{\star}^{\Omega}}{\delta \phi}=0 \Leftrightarrow & -\partial_{\rho} \partial^{\rho} \bar{\phi}+m^{2} \bar{\phi}+\frac{\lambda}{2.4 !}(2 \bar{\phi} \star \phi \star \bar{\phi}+\bar{\phi} \star \bar{\phi} \star \phi+\phi \star \bar{\phi} \star \bar{\phi}) \\
& +\frac{\Omega^{2}}{8}(2 \tilde{x} \star \bar{\phi} \star \tilde{x}+\bar{\phi} \star \tilde{x} \star \tilde{x}+\tilde{x} \star \tilde{x} \star \bar{\phi})=0, \\
\frac{\delta S_{\star}^{\Omega}}{\delta \bar{\phi}}=0 \Leftrightarrow \quad- & \partial_{\rho} \partial^{\rho} \phi+m^{2} \phi+\frac{\lambda}{2.4 !}(2 \phi \star \bar{\phi} \star \phi+\phi \star \phi \star \bar{\phi}+\bar{\phi} \star \phi \star \phi) \\
& +\frac{\Omega^{2}}{8}(2 \tilde{x} \star \phi \star \tilde{x}+\phi \star \tilde{x} \star \tilde{x}+\tilde{x} \star \tilde{x} \star \phi)=0 .
\end{aligned}
$$

One avoids translational invariance violation for the appearance of the coordinate $\widetilde{x}^{\rho}$ by considering further the constraint $\delta S_{\star}^{\Omega} / \delta \tilde{x}_{\rho}=0$, i.e.

$$
\begin{aligned}
& \frac{\Omega^{2}}{8}\left(2 \phi \star \tilde{x}^{\rho} \star \bar{\phi}+2 \bar{\phi} \star \tilde{x}^{\rho} \star \phi+\phi \star \bar{\phi} \star \tilde{x}^{\rho}\right. \\
+ & \left.\tilde{x}^{\rho} \star \phi \star \bar{\phi}+\tilde{x}^{\rho} \star \bar{\phi} \star \phi+\bar{\phi} \star \phi \star \tilde{x}^{\rho}\right)=0 .
\end{aligned}
$$

From infinitesimal translations, we can now define the global canonical Ward identity operator for the NC complex GW model

$$
\begin{aligned}
\mathcal{W}_{\mu}^{\theta} & =\int d^{D} x\left(\partial_{\mu} \phi \star \frac{\delta}{\delta \phi}+\frac{\delta}{\delta \phi} \star \partial_{\mu} \phi+\partial_{\mu} \bar{\phi} \star \frac{\delta}{\delta \bar{\phi}}+\frac{\delta}{\delta \bar{\phi}} \star \partial_{\mu} \bar{\phi}\right. \\
& \left.+\partial_{\mu} \tilde{x}_{\rho} \star \frac{\delta}{\delta \tilde{x}_{\rho}}+\frac{\delta}{\delta \tilde{x}_{\rho}} \star \partial_{\mu} \tilde{x}_{\rho}\right)
\end{aligned}
$$

such that its action on the Lagrangian density

$$
\mathcal{W}_{\mu}^{\theta} S_{\star}^{\Omega} \equiv-\int d^{D} x \partial^{\rho} T_{\rho \mu}^{\Omega}=0
$$

gives the canonical energy momentum tensor (EMT)

$$
T_{\rho \mu}^{\Omega}=\frac{1}{2}\left\{\partial_{\mu} \phi, \partial_{\rho} \bar{\phi}\right\}_{\star}+\frac{1}{2}\left\{\partial_{\mu} \bar{\phi}, \partial_{\rho} \phi\right\}_{\star}-g_{\rho \mu} \mathcal{L}_{\star}^{\Omega},
$$

where $g_{\rho \mu}$ is the Euclidean metric, $\mathcal{L}_{\star}^{\Omega}$ the NC Lagrangian, $\{(\cdot),(\cdot)\}_{\star}$ the $\star$-anticommutator. The EMT then conserves its form comparatively to the result of [10]. $T_{\rho \mu}^{\Omega}$ is symmetric, nonlocally conserved, and in massless theory, not traceless. Moreover, putting the mass term to zero, the usual improved tensor

$$
T_{\rho \mu}^{I, \Omega}=T_{\rho \mu}^{\Omega, m=o}+\frac{1}{6}\left(g_{\rho \mu} \square^{-} \partial_{\rho} \partial_{\mu}\right)\{\phi, \bar{\phi}\}_{\star}
$$

is not traceless too. Let us investigate now an improvement to (2.5) for the local conservation order. After some algebra, it can be deduced

$$
\begin{aligned}
\partial^{\rho} T_{\rho \mu}^{\Omega} & =\partial^{\rho} T_{\rho \mu}-\frac{\Omega^{2}}{16}\left(\left[\tilde{x} \star \tilde{x}, \partial_{\mu} \phi \star \bar{\phi}+\partial_{\mu} \bar{\phi} \star \phi\right]_{\star}+\left[\tilde{x} \star \bar{\phi}, \partial_{\mu} \phi \star \tilde{x}\right]_{\star}\right. \\
& \left.+\left[\tilde{x} \star \phi, \partial_{\mu} \bar{\phi} \star \tilde{x}\right]_{\star}+\left[\tilde{x} \star \partial_{\mu} \phi, \bar{\phi} \star \tilde{x}\right]_{\star}+\left[\tilde{x} \star \partial_{\mu} \bar{\phi}, \phi \star \tilde{x}\right]_{\star}\right) \\
& -\frac{\Omega^{2}}{8}\left[\tilde{x}, \partial_{\mu} \phi \star \tilde{x} \star \bar{\phi}+\partial_{\mu} \bar{\phi} \star \tilde{x} \star \phi\right]_{\star}
\end{aligned}
$$




$$
=: \quad-\partial^{\rho} t_{\rho \mu}^{\Omega}
$$

where

$$
\begin{aligned}
\partial^{\rho} T_{\rho \mu} & =-\frac{\lambda}{4(4 !)}\left(\left[\phi \star \bar{\phi}, \partial_{\mu} \phi \star \bar{\phi}-\phi \star \partial_{\mu} \bar{\phi}\right]_{\star}+\left[\bar{\phi} \star \phi, \partial_{\mu} \bar{\phi} \star \phi-\bar{\phi} \star \partial_{\mu} \phi\right]_{\star}\right. \\
& +\frac{1}{2}\left[\phi \star \bar{\phi}, \partial_{\mu} \bar{\phi} \star \phi-\bar{\phi} \star \partial_{\mu} \phi\right]_{\star}+\frac{1}{2}\left[\bar{\phi} \star \phi, \partial_{\mu} \phi \star \bar{\phi}-\phi \star \partial_{\mu} \bar{\phi}\right]_{\star} \\
& \left.+\frac{1}{2}\left[\phi \star \phi,\left[\partial_{\mu} \bar{\phi}, \bar{\phi}\right]_{\star}\right]_{\star}+\frac{1}{2}\left[\bar{\phi} \star \bar{\phi},\left[\partial_{\mu} \phi, \phi\right]_{\star}\right]_{\star}\right) .
\end{aligned}
$$

$[(\cdot),(\cdot)]_{\star}=$ Moyal $\star$-commutator. Thus the "Wulkenization" 14 process clearly governs the EMT improvement mechanism. A closer look on (2.6) shows that $T_{\rho \mu}^{\Omega}$ is globally conserved, as in NCFT the $\star$-commutators under integral cancel. For physical interpretation, let us consider the space-space NCFT determined by $\Theta^{0 i}=0$. Then one can readily prove that there exists a conserved $D$-vector momentum $P_{\mu}^{\Omega}$, namely $\partial^{0} P_{\mu}^{\Omega}=\partial^{0} \int d^{D-1} x T_{0 \mu}^{\Omega}=0$. Such a vector conserved quantity is also observed in the naive (unrenormalizable) NC scalar field [2] as well as in the NC real GW model [10]. Besides, it turns out that, displaying the same tedious algebraic apparatus as in 10, a correction term can be provided to get a new locally conserved albeit nonsymmetric EMT, $\hat{T}_{\rho \mu}^{I, \Omega}=T_{\rho \mu}^{I, \Omega}+t_{\rho \mu}^{\Omega}$, with nonvanishing trace for $\mathrm{m}=0$ recalling that the theory is not scale invariant. Furtheremore, one can work out a symmetric locally conserved EMT through the ordinary Belifante trick (see [10] and references therein), defining the tensor $\chi_{\sigma \rho \mu}$ such that

$$
\hat{T}_{\rho \mu}^{\Omega s}=\hat{T}_{\rho \mu}^{\Omega}+\partial^{\sigma} \chi_{\sigma \rho \mu}, \quad \chi_{\sigma \rho \mu}=-\chi_{\rho \sigma \mu} .
$$

The underlying Belifante type partial differential equation

$$
\hat{T}_{\rho \mu}^{\Omega}-\hat{T}_{\mu \rho}^{\Omega}=\partial^{\sigma}\left(\chi_{\sigma \mu \rho}-\chi_{\sigma \rho \mu}\right)=: \partial^{\sigma} \chi_{\sigma[\mu \rho]}
$$

is less comfortable than the one worked out by Abou-Zeid and Dorn [3]. More detailed consideration of various properties of EMTS will appear elsewhere.

Let us now better scrutinize the nonlocal conservation of the canonical massless EMT. It obviously induces a dilatation symmetry breaking. In addition, even if an improved locally conserved EMT is provided, the scale invariance is no longer valid and predictable since the evidence of a nonvanishing trace of this improved EMT. Both these arguments on dilatation symmetry breaking are valid for massless Grosse and Wulkenhaar model. Indeed, defining infinitesimal dilatation generators and the corresponding global symmetrized Wlop $W_{D, \epsilon}^{\theta}$, respectively,

$$
\begin{aligned}
\delta_{\epsilon} \tilde{x}_{\mu} & =(1+\epsilon) \tilde{x}_{\mu}, \quad \delta_{1, \epsilon} \phi=\epsilon D_{1} \phi, \quad \delta_{2, \epsilon} \phi=\epsilon D_{2} \phi \\
D_{1}(.) & =\left(1+x^{\mu} \star \partial_{\mu}\right)(.), \quad D_{2}(.)=\left((.)+\partial_{\mu}(.) \star x^{\mu}\right) \\
W_{D, \epsilon}^{\theta}(.) & =\int d^{D} x\left\{\frac { \epsilon } { 4 } \left[D_{1} \phi \star \frac{\delta(.)}{\delta \phi}+\frac{\delta(.)}{\delta \phi} \star D_{1} \phi+D_{2} \phi \star \frac{\delta(.)}{\delta \phi}+\frac{\delta(.)}{\delta \phi} \star D_{2} \phi\right.\right. \\
+ & \left.(\phi \longleftrightarrow \bar{\phi})]+\frac{1}{2}\left[\delta_{\epsilon} \tilde{x}_{\rho} \star \frac{\delta(.)}{\partial \tilde{x}_{\rho}}+\frac{\delta(.)}{\delta \tilde{x}_{\rho}} \star \delta_{\epsilon} \tilde{x}_{\rho}\right]\right\}
\end{aligned}
$$


so that $\frac{\partial}{\partial \epsilon} W_{D, \epsilon}^{\theta}\left(S_{\star}^{\Omega}\right)=-\int d^{D} x\left(\partial^{\rho} \mathcal{D}_{\rho}^{\Omega}+B_{\star}^{\Omega}\right)$ with $\mathcal{D}_{\rho}^{\Omega}$ the dilatation current given by $\mathcal{D}_{\rho}^{\Omega}=\frac{1}{2}\left\{x^{\mu}, \hat{T}_{\rho \mu}^{I, \Omega}\right\}_{\star}$, the breaking quantity $B_{\star}^{\Omega}$ reveals to depend both on the nonvanishing trace of the local conservation improving tensor $t_{\rho \mu}^{\Omega}$ through $\hat{T}_{\mu}^{I, \Omega, \mu}$ and on the GW term as follows:

$$
\begin{aligned}
& B_{\star}^{\Omega}=-\hat{T}_{\mu}^{I, \Omega, \mu}-\frac{1}{2}\left\{x^{\mu}, \frac{\lambda}{2 .(4 !)}\left[\partial_{\mu} \phi \star\left(2 \bar{\phi} \star \phi \star \bar{\phi}+\{\bar{\phi} \star \bar{\phi}, \phi\}_{\star}\right)\right.\right. \\
& +\partial_{\mu} \bar{\phi}\left(2 \phi \star \bar{\phi} \star \phi+\{\bar{\phi}, \phi \star \phi\}_{\star}\right)-\frac{1}{2} \partial_{\mu}\left(\{\phi \star \bar{\phi} \star \phi, \bar{\phi}\}_{\star}\right) \\
& \left.+\frac{1}{4} \partial_{\mu}\left(\{\phi \star \bar{\phi}, \bar{\phi} \star \phi\}_{\star}+\{\phi \star \phi, \bar{\phi} \star \bar{\phi}\}_{\star}\right)\right]+\frac{\Omega^{2}}{8}\left[-\partial_{\mu}\left(\{\tilde{x} \star \phi, \tilde{x} \star \bar{\phi}\}_{\star}\right.\right. \\
& \left.+\frac{1}{2}\left\{\tilde{x} \star\{\bar{\phi}, \phi\}_{\star}, \tilde{x}\right\}_{\star}\right)+\partial_{\mu} \phi \star\left(2 \tilde{x} \star \bar{\phi} \star \tilde{x}+\{\bar{\phi}, \tilde{x} \star \tilde{x}\}_{\star}\right) \\
& \left.\left.+\partial_{\mu} \bar{\phi} \star\left(2 \tilde{x} \star \phi \star \tilde{x}+\{\phi, \tilde{x} \star \tilde{x}\}_{\star}\right)\right]\right\}_{\star}
\end{aligned}
$$

Finally, let $U_{\star}(1)$ be the NC gauge group generated by elements $U \in$ $U_{\star}(1)$ :

$$
e_{\star}^{i \alpha}=1+i \alpha+\left(i^{2} / 2 !\right) \alpha \star \alpha+\left(i^{3} / 3 !\right) \alpha \star \alpha \star \alpha+\ldots, \alpha \in C^{\infty}(\mathbf{R})
$$

Then, defining the left group action and infinitesimal transformations

$$
\phi \longmapsto \phi^{\prime}=U_{\star} \phi ; \quad \delta_{\alpha} \phi=i \alpha \star \phi ; \quad \bar{\phi} \longmapsto \bar{\phi}^{\prime}=\bar{\phi} U_{\star}^{\dagger} ; \quad \delta_{\alpha} \bar{\phi}=-i \bar{\phi} \star \alpha,
$$

and the infinitesimal action variation

$$
\delta S=\int\left\{\partial_{\mu}\left(\delta \phi \star \partial^{\mu} \bar{\phi}\right)+\partial_{\mu}\left(\partial^{\mu} \phi \star \delta \bar{\phi}\right)+\delta \bar{\phi} \star(\mathcal{E} \mathcal{L}(\phi))+(\mathcal{E} \mathcal{L}(\bar{\phi})) \star \delta \phi\right\}(2.11
$$

where $\mathcal{E} \mathcal{L}(\phi)$ (resp. $\mathcal{E} \mathcal{L}(\bar{\phi})$ ) represents the Euler-Lagrange equations of motion for $\phi$ (resp. for $\bar{\phi}$ ), leads to express $U_{\star}(1)$ gauge WIop in the form

$$
W^{G}(\cdot)=\int d^{D} y \frac{\delta}{\delta \alpha(y)} \int d^{D} x\left[\frac{\delta(\cdot)}{\delta \bar{\phi}} \star \delta \phi+\delta \bar{\phi} \star \frac{\delta(\cdot)}{\delta \phi}\right]
$$

such that $W^{G}(S)=\int d^{D} y \partial_{\mu} J^{\mu}$ yields the suitable NC $U_{\star}(1)$ gauge currents of the GW model as follows:

$$
J^{\mu}=i\left(\phi \star \partial^{\mu} \bar{\phi}-\partial^{\mu} \phi \star \bar{\phi}\right) .
$$

\section{Conclusion}

This work has proved that the WIop formulation of the NC Noether theorem given in 11 is one of the keystones in Moyal field theory analysis. Its successful application here allowed to investigate Neither currents for NC complex GW model. Symmetry properties (translation, dilatation and gauge symmetries) have been studied. EMTs are regularizable by usual methods. $U_{\star}(1)$ gauge currents have been explicitly computed. 


\section{Acknowledgment}

This work is partially supported by the ICTP through the OEA-ICMPAPrj-15. The ICMPA is in partnership with the Daniel Iagolnitzer Foundation (DIF), France. The authors thank Dr J. Ben Geloun for fruitful discussions. MNH expresses his gratefulness to Professor A. Odzijewicz and all his staff for their hospitality and the good organization of the Workshops in Geometric Methods in Physics.

\section{References}

[1] M. R. Douglas and N. A. Nekrasov, Noncommutative field theory, Rev. Mod. Phys. 73, 977-1029 (2001); [e-print hep-th/0106048]. R. J. Szabo, Quantum Field Theory on Noncommutative Spaces, Phys. Rept. 378, 207-299 (2003); [eprint hep-th/0109162.

[2] A. Gerhold, J. Grimstrup, H. Grosse, L. Popp, M. Schweda and R. Wulkenhaar, The energy momentum tensor on noncommutative spaces - Some pedagogical comments, unpublished, [e-print hep-th/0012112].

[3] M. Abou-Zeid and H. Dorn, Comments on the energy momentum tensor in noncommutative field theories, Phys. Lett. B 514, 193-188 (2001); [e-print hep-th/0104244].

[4] J. M. Grimstrup, B. Kloiböck, L. Popp, V. Putz, M. Schweda and M. Wickenhauser, The energy momentum tensor in noncommutative gauge field models, Int. J. Mod. Phys. A 19, 5615-5624 (2004); [e-print hep-th/0210288].

[5] A. Das and J. Frenkel, On the energy momentum tensor in noncommutative field theories, Phys. Rev. D 67, 067701 (2003); [e-print hep-th/0212122].

[6] M. N. Hounkonnou, F. Massamba and J. Ben Geloun, 2-dimensional noncommutative field theory on the light cone, JGSP 4, 38-46 (2005).

[7] J. Wess, Deformed coordinate spaces, derivatives, unpublished, [e-print hep-th/0408080; ibid, Deformed gauge theories, unpublished, [e-print hep-th/0608135.

[8] M. Chaichian, P. P. Kulish, K. Nishijima and A. Tureanu, On a LorentzInvariant Interpretation of Noncommutative Space-Time and Its Implications on Noncommutative QFT, Phys. Lett. B 604, 98-102 (2004); [e-print hep-th/0408069. F. Koch and E. Tsouchnika, Construction of $\theta$-Poincaré algebras and their invariants on $M_{\theta}$, Nucl. Phys. B 717, 387-403 (2005); [e-print hep-th/0409012. J. Lukierski, A. Nowicki and H. Ruegg, Phys. Lett. B 293, 344-352 (1992).

[9] H. Grosse and R. Wulkenhaar, Renormalization of $\phi^{4}$-theory on Non Commutative $\mathrm{R}^{4}$ in the matrix base, Comm. Math. Phys. 256, 305-374 (2005); [e-print hep-th/0401128.

[10] J. Ben Geloun and M. N. Hounkonnou, Energy-momentum tensors in renormalizable noncommutative scalar field theory, Physics Letters B 653 343-345 (2007).

[11] J. Ben Geloun and M. N. Hounkonnou, Noncommutative Noether theorem, AIP Proc 956 55-60 (2007). 
[12] J. Ben Geloun and M. N. Hounkonnou, Noncommutative Noether currents, (2007) in progress.

[13] J. M. Gracia-Bondia and J. C. Varilly, Algebras of distributions suitable for phase-space quantum mécanics I, J. Math. Phys. 29, 869-879 (1988).

[14] V. Rivasseau, Noncommutative renormalization, Seminaire Poincaré X Espace Quantique, Inst. Henri Poincaré, Paris, 2007, pp 15-95.

[15] E. Langmann and R. J. Szabo, Duality in scalar field theory on noncommutative phase spaces, Phys. Lett. B 533, 168-177 (2002); [e-print hep-th/0202039].

Mahouton Norbert Hounkonnou

University of Abomey-Calavi

International Chair in Mathematical Physics and Applications

ICMPA-UNESCO CHAIR

072B.P.:50, Cotonou, Rep. of Benin

e-mail: norbert.hounkonnou@cipma.uac.bj with copy to hounkonnou@yahoo.fr

Dine Ousmane Samary

University of Abomey-Calavi

International Chair in Mathematical Physics and Applications

ICMPA-UNESCO CHAIR

072B.P.:50, Cotonou, Rep. of Benin

e-mail: ousmanesamarydine@yahoo.fr 